\title{
Informational Framework for Minimalistic Visual Odometry on Outdoor Robot
}

\author{
Joelle Al Hage ${ }^{12}$, Stefano Mafrica ${ }^{3}{ }^{4}$, Maan E.El Najjar ${ }^{1}$, Franck Ruffier ${ }^{3}$
}

\begin{abstract}
In an unknown environment, assessing the robot trajectory in real time is one of the key issues for a successful robotic mission. In such environment, the absolute measurements like the GPS data may be unavailable. Moreover, estimating the position using only proprioceptive sensors like encoders and Inertial Measurements Units (IMU) will generate errors that increase over time. This paper presents a multi-sensor fusion approach between IMU and ground Optical Flow (OF) used to estimate the position of a mobile robot while ensuring high integrity localization. The data fusion in done through the informational form of the Kalman Filter namely Information Filter (IF). A Fault Detection and Exclusion (FDE) step is added in order to exclude the erroneous measurements from the fusion procedure by making it fault tolerant and to ensure a high localization performance. The approach is based on the use of the IF for the state estimation and tools from the information theory for the FDE. Our proposed approach evaluates the quality of a measurement based on the amount of information it provides using informational metrics like the Kullback-Leibler divergence. The approach is validated on data obtained from experiments performed in outdoor environments in various conditions including high-dynamic-range lighting and different ground textures.
\end{abstract}

Index Terms-Mobile Robot, Odometry, Data fusion, Optic Flow, Information Filter, Kullback-Leibler Divergence, Fault Detection and Exclusion

\section{INTRODUCTION}

$\mathrm{A}$ CCURATE positioning estimations are required for successful operation when navigating in an unknown environment or near humans. Multi-sensor data fusion has been addressed in various research [1] thanks to its advantages involving enhancements in the measurement availability and the integrity of estimation. However, when fusing measurements from multiple sources some problems may arise such as data imperfections and measurement errors. These problems may lead to an inaccurate estimation implying the necessity of the exclusion of the erroneous data from the fusion procedure [2] [3].

1 CRIStAL laboratory CNRS UMR 9189, University of Lille, 59650 Villeneuve d'Ascq, France

${ }^{2}$ Heudiasyc laboratory CNRS UMR 7253, Université de Technologie de Compiègne, 60200, Compiègne, France.

${ }^{3}$ Aix Marseille Univ, CNRS, ISM, Marseille 13009, France

${ }^{4}$ PSA Peugeot Citroën, 78140 Vélizy-Villacoublay, France

joelle.al-hage@hds.utc.fr, stefano.mafrica@mpsa.com,

maan.e-elnajjar@univ-lille1.fr, franck.ruffier@univ-amu.fr
Visual odometry techniques based on motion tracking between two images have proved their efficiency for localizing robots during planetary operations [4] [5], underwater mission [6] or on-road navigation [7]. New approaches in visual odometry used different methods to be robust to various and poor light levels such as event-based techniques [8],[9] or binary descriptors [10]. However, solutions based on the use of a standard camera have disadvantages such as the high computational cost of the image processing, their expensiveness for robotic applications and their inability to cope with a large dynamic range of light conditions. In order to deal with the problems encountered with the standard camera, low-cost Optical Flow (OF) sensors have been developed in various researches. In [11], solution based on optical mince is proposed. The sensors provide highfrequency data and they are characterized by an extremely low cost. However, their main drawback is that they should operate near the ground and they are unsuitable for rough terrain. In [12], solution based on Optical sensors and Laser sources is developed to operate on different surface materials. In [13], a minimalistic bio-inspired OF sensors are presented. Experiments in indoor and outdoor environments show that the optic flow measurements are robust to high-dynamic-range lighting conditions and to various ground textures. These sensors developed in the ISM laboratory (Marseilles, France) are used in this paper in order to perform a visual guidance.

The Inertial Measurement Unit (IMU) is an electronic system consisting of accelerometers and gyroscopes. In the odometry context, the mathematical integration of these sensors outputs suffer from accumulated errors leading to a drift over time. On the other hand, the OF measurements may be unavailable on some trajectories parts. In particular, an outdoor terrain is not completely slick; an EKF solely based optic flow or solely based on IMU will be therefore affected by some vibrations [13] (figure 1c). As a consequence, a minimalistic visual odometry based on a unique sensor type will drift dramatically. In order to face the restriction of each sensor type, in this work, we propose to fuse the OF with the IMU measurements. This fusion is done through the informational form of the Kalman Filter (KF). Indeed, the efficiency of the Information Filter (IF) [14] has been proven for multi-sensor data fusion [15] where a simplified expression of the update step is obtained after dealing with the information vector and the information matrix. This 
expression is modelled as a simple sum of the information contributions of the different measurements. The update step of the IF lends itself well to real-time applications, especially when the number of measurements is greater than the dimension of the state vector as well for the FDE step.

Fault tolerant data fusion has been evoked in the literature in various researches. In [16], the authors proposed a method for detecting and isolating the sensors failures based on a bank of KF. Each filter represents a specific failure model and produces a specific residual. In [17], a method for detecting and excluding the erroneous GPS measurements by using a bank of KF is proposed. This method is widely used in the field of GNSS to ensure the integrity of localization. Initially, an estimation of the position obtained using the main $\mathrm{KF}$ taking into account $N$ satellites measurements is carried out in order to be compared with estimates obtained from a bank of filter each incorporating $N-1$ measurements. For the exclusion of faults, another bank of filter integrating $N-2$ measurements must be established. This method suffers from a high computational cost especially in the case of multiple faults isolation. In [18], two methods based on the use of the particular filter are proposed in order to deal with the sensors' measurements validation for aerial application. It is a method based on model with interaction between the models. In [19], an approach that uses the Bayesian inference permits to identify automatically the inconsistent data and to isolate them from the fusion procedure. The method adds a term to the Bayesian formulation which is the estimate of the probability in the case of normal operation. An inconsistent sensor increases the variance of the posterior distribution. In [3], the authors proposed a method of fusion and validation of the sensors based on the Nadaraya-Watson statistic. This is a method which requires a priori information on the sensors in the case of their normal operation. The faulty sensor will be detected and isolated from the fusion procedure. In [20], the method is based on hardware redundancy and on the analysis of conflicts between the data sources found on transferable beliefs. A source is considered to be faulty if its conflict is high with the others. After detecting the faulty source, its effect on the final decision will be reduced without the necessity of its exclusion from the fusion procedure.

These methods found in the literature require a priori knowledge on the fault models, information on the sensors in their normal operation, expertise, or availability of sufficient data. In this work, a method for fault tolerant data fusion is developed. It is based on the use of a general information framework which takes benefit of the informational form of the KF and tools from the information theory. Residual obtained from the Kullback-Leibler Divergence (KLD) between the a priori and a posteriori distributions of the Extended IF (EIF) allows the detection of faults. This residual incorporates two terms: Mahalanobis distance which permits a test on the means of the data distributions and Burg matrix divergence which permits a test on the covariance matrix. After detecting a fault, a bank of EIF is developed and a set of residual based on the KLD is generated. Each residual takes into account the information contribution of one measurement (named also observation from a state space representation point of view). The measurement that leads to the maximum value of the KLD will be excluded from the fusion procedure. Our approach evaluates the quantity of information provided by a sensor measurement which permits to evaluate the quality of this measurement; this is relevant from the fault tolerance point of view.

The main contributions of this paper are:

- Data fusion between IMU and OF measurements for ensuring high integrity localization of a mobile robot

- The use of the information filter for the data fusion and the KLD for the fault detection and exclusion

- Experimental study of the developed method in outdoor environment in various conditions including high-dynamic-range lighting and different ground textures.

- Comparison with previous results based on the KF and the median filter.

The paper is organised as follows: the fusion between the $\mathrm{OF}$ and the IMU measurements through the EIF is presented in Section 2. The informational framework for the FDE step is detailed in section 3. Experimental results carried on a car-like robot in outdoor environments with comparison with previous results obtained using the KF are detailed in section 4, followed by the authors' conclusions in section 5 .

\section{INFORMATION FILTER FOR IMU/OPTIC FLOW DATA FUSION}

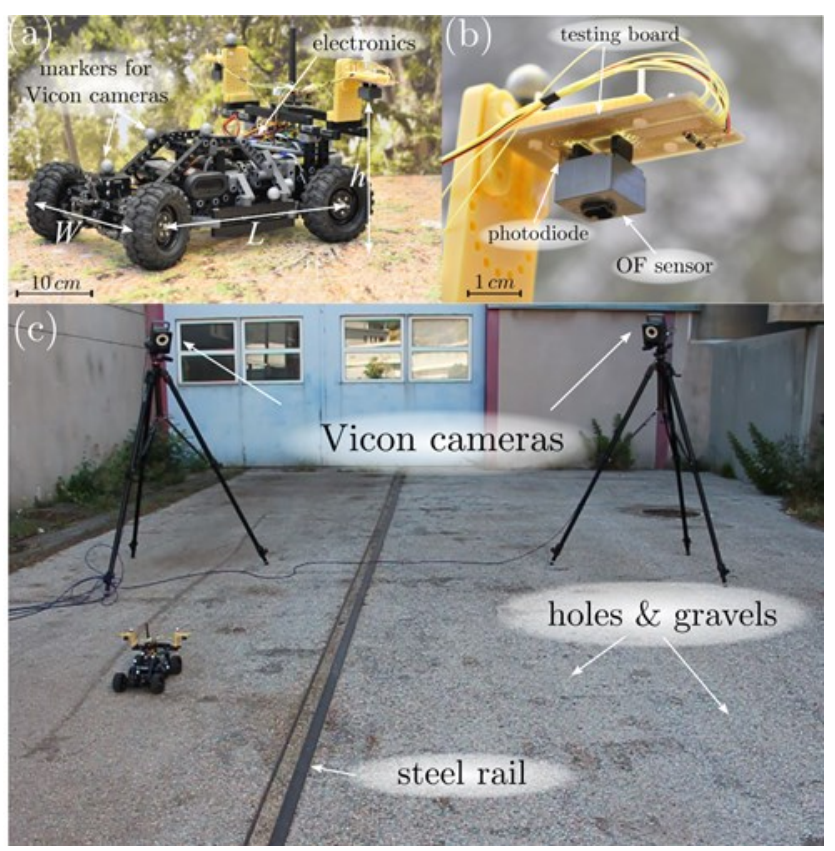

Fig. 1. (a) The low-cost car-like robot called BioCarBot. (b) One of the two OF sensors used on the robot. (c) The outdoor test environment equipped with Vicon cameras. The ground, which consisted mainly of asphalt, included holes, gravel and a disturbing steel rail. Adapted from [13].

In this study, we focused on a car-like robot (Figure 1) driven by a DC motor and equipped with two downwardfacing 12-pixel Optic Flow (OF) sensors [13] (one on the left 
and the other on the right) as well an IMU. This robot estimates its own velocity and steering angle, and therefore its position and orientation, based on the following informational approach.

The state vector of the robot is taken to be the longitudinal velocity $V$ and the steering angle $\emptyset: \xi=[V, \varnothing]^{T}$. In order to estimate the robot's state vector, an Extended Information Filter (EIF) is applied which is composed of two steps:

- Prediction step where the dynamics of $V$ and $\varnothing$ are obtained from a model (previously identified using the ground-truth measurements),

- update step where the OF and the IMU measurements are used in order to correct the prediction.

\section{A. Prediction step}

The dynamics of $V$ and $\varnothing$ are identified in the following form [13]:

$$
\xi_{k+1}=\xi_{k}+\left[A \xi_{k}+B u_{k}\right] T_{e}
$$

where:

$\xi_{k}$ denotes the state vector at the discretization time $k$

$A=\operatorname{diag}\left(a_{1}, a_{2}\right), B=\operatorname{diag}\left(b_{1}, b_{2}\right)$

$a_{1}=-b_{1}=-2.15, a_{2}=-b_{2}=-4.87$

$u_{k}$ is the input vector obtained from the control inputs at instant $k$

$T_{e}$ is the sampling period.

The covariance matrix is written as follows:

$$
P_{k+1 / k}=F_{k} P_{k / k} F_{k}^{T}+Q
$$

where:

$$
F_{k}=I_{2 \times 2}+A T_{e}
$$

and $Q$ is the covariance matrix associated to the process state noise, which is supposed to be white Gaussian noise with a zero mean.

Since the estimator is the EIF, the information matrix and the information vector have to be calculated: these are expressed in equations (4) and (5), respectively [15]:

$$
\begin{gathered}
Y_{k+1 / k}=P_{k+1 / k}^{-1} \\
y_{k+1 / k}=Y_{k+1 / k} \xi_{k+1 / k}
\end{gathered}
$$

\section{B. Update step}

In order to update the predicted estimation, the OF and the IMU measurements are fused through the EIF.

The OF measured between the $(i-1)$-th and the $i$-th pixels is denoted by $\omega_{i}$ and modelled as follows:

$$
z_{i}=\left(\begin{array}{c}
\omega_{i}^{l} \\
\omega_{i}^{r}
\end{array}\right)=h_{i}(k)=\left(\begin{array}{c}
\frac{\left(L-y_{l} \tan \emptyset\right) \sin ^{2} \varphi_{i}^{l}}{h L} \\
\frac{\left(L-y_{r} \tan \emptyset\right) \sin ^{2} \varphi_{i}^{r}}{h L}
\end{array}\right) V
$$

where:

$\omega_{i}^{l}$ and $\omega_{i}^{r}$ are the $i$-th OFs delivered by the left and right sensors, respectively, $\varphi_{i}^{l}$ and $\varphi_{i}^{r}$ are the orientations of the pixel's axis corresponding to the OF values $\omega_{i}^{l}$ and $\omega_{i}^{r}$.

$L(=255 \mathrm{~mm})$ is the distance between the rear and front wheel axes,

$y_{l}=-y_{r}(=140 \mathrm{~mm})$ are the $y$-values of the left and right OF sensors with respect to the body frame $<\mathrm{B}>$ placed in the middle of the rear wheel axis.

$h$ is the height of the OF sensors above the ground.

Since the observation model is non-linear, the EIF is applied and the Jacobian matrix $H_{i}=\left.\frac{\partial h_{i}}{\partial \xi}\right|_{\xi_{k / k-1}}$ is computed:

$H_{i}=$

$$
\left(\begin{array}{ll}
\frac{\left(L-y_{l} \tan \emptyset\right) \sin ^{2} \varphi_{i}^{l}}{h L} & \frac{-y_{l} \sin ^{2} \varphi_{i}^{l}\left(1+\tan ^{2} \emptyset\right) V}{h L} \\
\frac{\left(L-y_{r} \tan \emptyset\right) \sin ^{2} \varphi_{i}^{r}}{h L} & \frac{-y_{r} \sin ^{2} \varphi_{i}^{r}\left(1+\tan ^{2} \emptyset\right) V}{h L}
\end{array}\right)_{k / k-1}
$$

The noise associated with the OF measurements is assumed to be white Gaussian noise with zero mean and covariance matrix $R=\operatorname{diag}\left(\sigma_{l}^{2}, \sigma_{r}^{2}\right)$.

On the other hand, the output model corresponding to the IMU measurements is given by:

$$
\begin{aligned}
Z_{I M U}=\left(\begin{array}{c}
a_{x} \\
\Omega_{z} \\
\Omega_{D C}
\end{array}\right)= & h_{I M U}(k) \\
& \approx\left(\begin{array}{c}
a_{1} V+b_{1} u_{1}+g \sin \beta_{I M U} \\
\frac{\tan \emptyset}{L} V \\
\frac{k_{g}}{2 \pi r} V
\end{array}\right)
\end{aligned}
$$

where $\Omega_{z}$ is the angular velocity obtained in terms of the Ackermann steering geometry [21], $a_{x}$ is the linear acceleration on the $x$-axis, $\Omega_{D C}$ is the DC motor's speed, $\mathrm{g}$ is the gravity acceleration, $r(=14 \mathrm{~mm})$ is the radius of the robot's wheel, $k_{g}(=3.4)$ is the transmission gear ratio and $\beta_{I M U}$ is the angle giving the rotation about the $y$-axis between the body frame $<\mathrm{B}>$ and the inertial frame $<\mathrm{I}>$. Note that in the inertial frame, the $x$ - and $y$-axes lie on the local ground plane (for further details, see [13]).

The observation matrix $H_{I M U}$ associated with the IMU observation model can be written as follows:

$H_{I M U}=$

$$
\left(\begin{array}{cc}
a_{1} & 0 \\
\frac{\tan \emptyset}{L} & \frac{V}{L}\left(1+\tan ^{2} \emptyset\right) \\
\frac{k_{g}}{2 \pi r} & 0
\end{array}\right)
$$

The noise associated with the IMU observations is assumed to be white Gaussian noise with a zero mean and covariance matrix $R_{I M U}=\operatorname{diag}\left(0.263^{2}, 0.047^{2}, 0.001^{2}\right)$.

The update of the information matrix and information vector are obtained as in equations (10) and (11) respectively:

$$
Y_{k / k}=Y_{k / k-1}+\sum_{j=1}^{N} I_{j}(k)
$$




$$
y_{k / k}=y_{k / k-1}+\sum_{j=1}^{N} i_{j}(k)
$$

where $N$ is the number of observations and $\left(I_{j}(k), i_{j}(k)\right)$ are the information contributions of the observation $z_{j}\left(z_{j}=\right.$ $\left[\omega_{j}^{l} \omega_{j}^{r}\right]^{T}$ or $\left.z_{j}=z_{I M U}\right)$ :

$$
\begin{gathered}
I_{j}(k)=H_{j, k}^{T} R_{j}^{-1}(k) H_{j, k} \\
i_{j}(k)=H_{j, k}^{T} R_{j}^{-1}(k)\left[\left(z_{j, k}-z_{j, \text { estimate }}\right)+H_{j, k} \xi_{k / k-1}\right]
\end{gathered}
$$

$Z_{j, \text { estimate }}$ and $H_{j, k}$ are given in equations (6) and (7), respectively, in the case of the OF measurements and in equations (8) and (9) in that of the IMU measurements.

It is worth noting that the main advantage of the EIF appears in the update step, where it is modelled as a simple sum of the information contributions of the various observations. This formula lends itself well to real time applications, especially when the number of observations is greater than the dimension of the state vector [22].

The absolute robot's position $([x, y])$ and orientation $\theta$ are obtained from the Ackermann model [21] using the EIF estimate $\xi_{k}$ as an input.

A summary of the multi-sensor data fusion is shown in figure 2 .

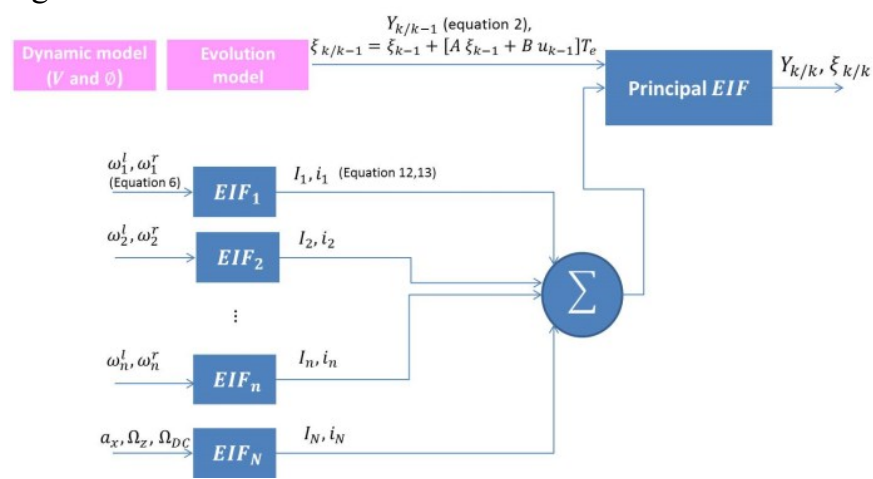

Fig. 2. A summary of the multi-sensor data fusion framework using the EIF

\section{Sensors Fault Detection And Exclusion Method BASED ON THE KULLBACK-LEIBLER DIVERGENCE}

The IMU and OF measurements could be affected by various errors that decrease the robot's localization integrity. These errors must therefore be detected and eliminated from the fusion procedure. In this paper, we propose an informational framework for the FDE based on the synthesis of a bank of EIF and residuals from the information theory.

The Kullback-Leibler Divergence (KLD) [23] [24] between two Gaussian distributions can take the form of a generalized residual. Indeed, the KLD between the a priori Gaussian probability distribution $(g(k / k-1))$ based on the dynamic model and a posteriori Gaussian probability distribution $(g(k / k))$ obtained from the update step of the EIF can be expressed as follows [15] :

$$
\begin{aligned}
G K L D & =K L D(g(k / k-1)|| g(k / k)) \\
& =\frac{1}{2} \operatorname{trace}\left(Y_{k / k} Y_{k / k-1}^{-1}\right)+\frac{1}{2} \log \frac{\left|Y_{k / k-1}\right|}{\left|Y_{k / k}\right|}-\frac{1}{2} M \\
& +\frac{1}{2}\left(\xi_{k / k}-\xi_{k / k-1}\right)^{T} Y_{k / k}\left(\xi_{k / k}-\xi_{k / k-1}\right)
\end{aligned}
$$

where $M$ is the dimension of the state vector $(M=2)$.

This residual namely the Global Kullback-Leibler Divergence $(G K L D)$ allows two types of tests [15]:

- Test on the means of the data distributions obtained using the Mahalanobis distances:

$\left(\xi_{k / k}-\xi_{k / k-1}\right)^{T} Y_{k / k}\left(\xi_{k / k}-\xi_{k / k-1}\right)$,

- Test on the covariance matrices obtained using Burg matrix divergence [25]:

$$
\operatorname{trace}\left(Y_{k / k} Y_{k / k-1}^{-1}\right)+\log \frac{\left|Y_{k / k-1}\right|}{\left|Y_{k / k}\right|}-M .
$$

When the $G K L D$ exceeds a threshold value, a sensor's fault could be detected. In the fault-free case, the general distribution of the $G K L D$ is related to the Chi-square and Fisher distributions [15] as follows:

$$
\begin{gathered}
G K L D \sim \frac{1}{2}\left[\frac{M(n-1)}{(n-M) n} F_{M, n-M}\right. \\
\left.+\frac{1}{n-1} \frac{1}{1-\frac{1}{6(n-1)-1}\left(2 M+1-\frac{2}{M+1}\right)} \chi_{\frac{1}{2}(M(M+1))}^{2}\right]
\end{gathered}
$$

where $n$ is the number of samples.

When a fault is detected using the GKLD, the erroneous measurements should be excluded from the fusion procedure. For this purpose, a bank of EIF is designed $\left(E I F_{j}\right)$ and a set of residual based on the KLD is developed. Each residual, denoted $K L^{j}$, takes into account the information contribution of one observation $z_{j}$ :

$$
\begin{aligned}
& K L_{k}^{j}= \\
& \quad \frac{1}{2} \operatorname{trace}\left(Y_{j k / k} Y_{k / k-1}^{-1}\right)+\frac{1}{2} \log \frac{\left|Y_{k / k-1}\right|}{\left|Y_{j k / k}\right|}-\frac{1}{2} M \\
& \quad+\frac{1}{2}\left[\left(\xi_{j k / k}-\xi_{k / k-1}\right)^{T} Y_{j k / k}\left(\xi_{j k / k}-\xi_{k / k-1}\right)\right]
\end{aligned}
$$

where:

$$
\begin{aligned}
Y_{j k / k} & =Y_{k / k-1}+I_{j}(k) \\
y_{j k / k} & =y_{k / k-1}+i_{j}(k) \\
\xi_{j k / k} & =\left(Y_{j k / k}\right)^{-1} y_{j k / k}
\end{aligned}
$$

$K L^{j}$ measures the divergence between the predicted distribution and the updated distribution obtained from a filter $E I F_{j}$ designed in such way that only the observation $z_{j}$ is taken into account. When the threshold is reached, the observation giving the maximum value of $K L^{j}$ is removed from the fusion procedure. This procedure is repeated iteratively until no faults are detected any longer using the $G K L D$ residual test (Figure $3)$.

Figure 3 presents a block diagram schema of the fault tolerant data fusion where a description is given in the following: using the dynamic model, the robot estimates its state vector and information matrix. Each filter $E I F_{j}$ computes the information contribution of one observation $Z_{j}$ and these 
contributions are sent to the main filter $E I F_{0}$ in order to update the predicted estimation. The output of this filter and the predicted distribution are used to calculate the $G K L D$. If a fault is detected, the set of residual $K L^{j}$ is generated in order to exclude the erroneous measurements from the fusion procedure and to re-estimate the state vector using the main filter.

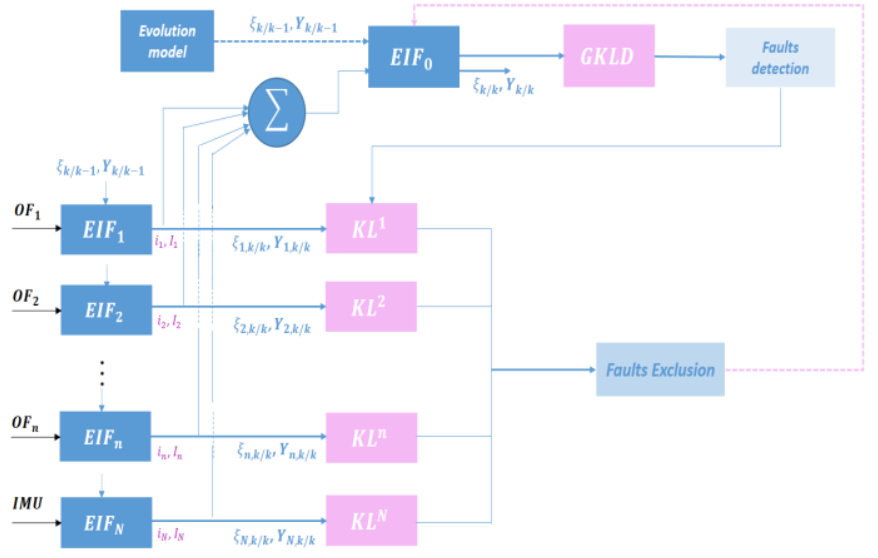

Fig. 3. The data fusion approach used here, including the FDE step

\section{EXPERIMENTS AND VALIDATION OF THE RESULTS}

Figure 1 shows pictures of the BioCarBot robot (Fig 1a), one of the two OF sensors used (also called VMSs [13],[26]) (Fig 1b) and the outdoor test environments (Fig 1c). The proposed approach is tested using a car-like robot based on the 2WD Racecar Kit provided by Minds-I Robotics, which is chosen despite the low resolution of the servo and motor control systems. The embedded electronics included one Nanowii board (Flyduino) and a MPU-6050 IMU comprising a 3-axis gyroscope and a 3-axis accelerometer (InvenSense) and one Overo IronSTORM computer-on-module (COM) (Gumstix). Two identical OF sensors giving ten 2-pixel raw downward-facing OF measurements on the right of the robot and ten 2-pixel raw downward-facing OF measurements on the left are attached to the robot's frame on both sides, aligned with the rear wheel axis (Figure 1a). To obtain ground-truth values, the robot's 3-D pose is measured with a Vicon motioncapture system via the infrared markers attached to the robot's frame (Figure 1a). Four individual Vicon cameras, each mounted on a tripod, are used in the outdoor experiments (Figures 1c). The robot is driven in a closed-loop mode based on the estimates of the velocity and steering angle values. IMU and OF sensors data are reused in batch mode to assess the robot's odometric performances based on the proposed informational framework.

Figure 4 shows the results obtained outdoor when the robot is driven on a circular path (Figure 4e) at constant steering angle and at velocity varying between 0.3 and $1.3 \mathrm{~m} / \mathrm{s}$ in presence of a recurrent vertical vibratory disturbance caused by a steel rail. (a)

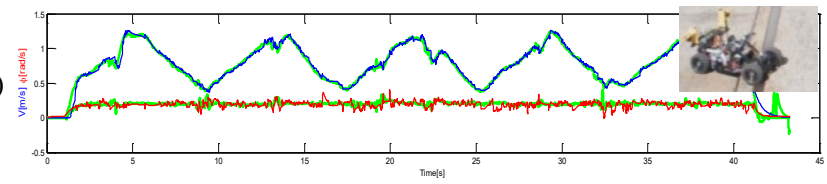

(b)

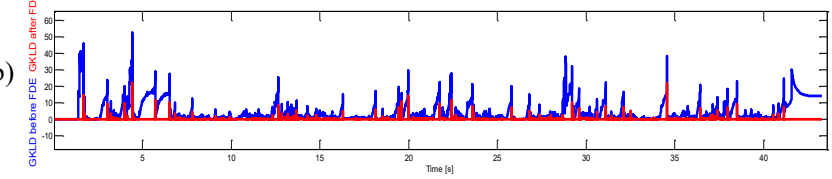

(c)

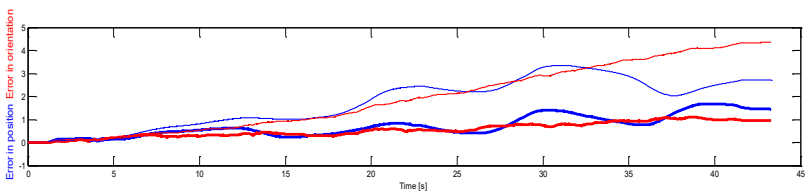

(d)

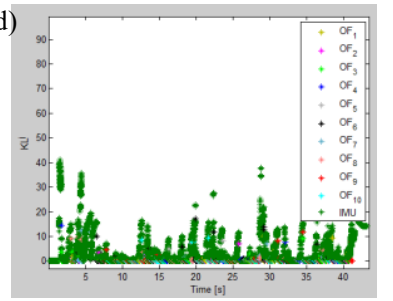

(e)

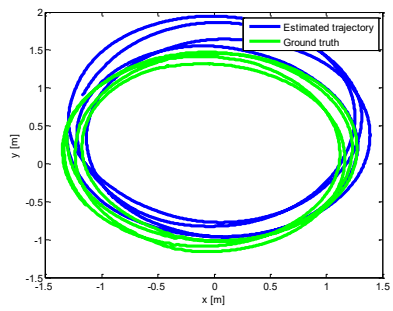

Fig. 4. Results obtained outdoors at very high light level in presence of recurrent vertically disturbance when the robot was driven on a circular path after fusing the IMU measurements with the OF data. (a) The estimated state vector after the FDE step (in blue and red), versus the ground truth values (in green). (Insert) Picture of the BioCarBot during the trial. (b) The GKLD residual used for the fault detection (before the FDE step in blue, after the FDE step in red). (c) The error in the absolute position and orientation estimation before the FDE step (light solid line) and after the FDE step (dark solid line). (d) The set of residuals used for the fault exclusion. (e) The estimated trajectory after the FDE step (in blue) compared to reference trajectory (in green).

Figure 4a shows the estimated longitudinal velocity $V$ and steering angle $\varnothing$ obtained from the fusion of the OF and IMU measurements through the EIF and after the FDE step. The $G K L D$ residual used to quantify the divergence between the predicted and updated distributions of the EIF is shown in Figure $4 \mathrm{~b}$. It presents many jumps indicating the presence of measurement errors. These errors should be excluded from the fusion procedure. For this purpose, the set of residuals $K L^{j}$ is calculated in order to quantify the information contributions of each measurement $z_{j}$ (Figure 4d). The measurement that leads to the maximum value of $K L^{j}$ is removed from the fusion procedure. The procedure is repeated iteratively until no fault is detected using the GKLD residual test. Notice, for this experiment, the errors appear especially at the level of the IMU measurements. Figure $4 \mathrm{c}$ shows the errors in position and orientation before (light solid line) and after the FDE step (dark solid line). This step therefore improved the error values, since the mean position error, which is about $1.7256 \mathrm{~m}$ before the FDE step, decreases to about $0.7169 \mathrm{~m}$ after the FDE step. Likewise, the mean orientation error, which is about $1.923 \mathrm{rad}$ before the FDE step, decreases to $0.5531 \mathrm{rad}$ after the FDE step. This experiment conducted in the outdoor environment in 
presence of vibratory perturbations proved the efficiency of the present informational framework as a means of detecting and excluding the faulty measurements from the fusion procedure, which greatly improved the robot's positioning performances. Likewise, this test shows the value of the OF sensors and their robustness to disturbances, which is greater than that of the IMU measurements.

(a)

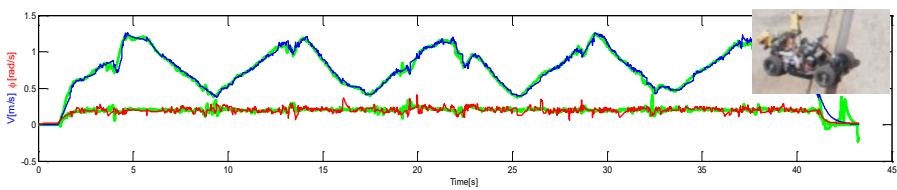

(b)

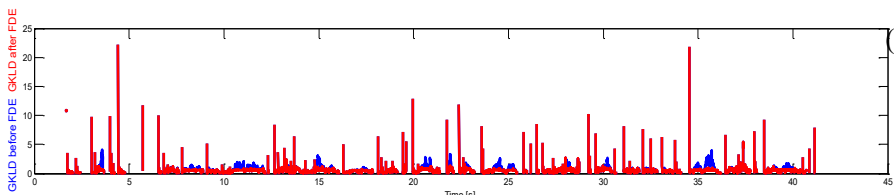

(c)

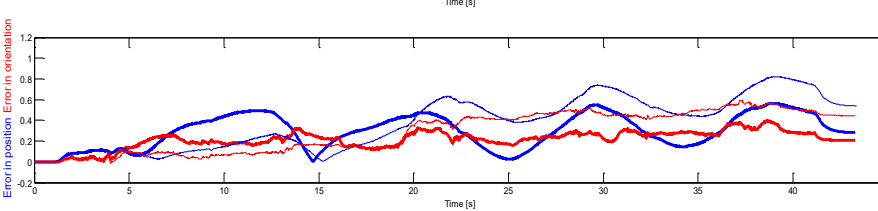

Fig. 5. Results without IMU obtained outdoors at very high light level when the robot was driven on a circular path in presence of recurrent vertically disturbance after fusing the OF raw data. (a) The estimated state vector after the FDE step (in blue and red), versus the ground truth values (in green). (Insert) Picture of the BioCarBot during the trial. (b) The GKLD residual used for the fault detection (before the FDE step in blue, after the FDE step in red). (c) The error in the absolute position and orientation estimation after the FDE step (dark solid line) compared to the ones obtained using the EKF and the OF median value (light solid line).

We compare in figure 5 the performance obtained by using the proposed informational framework to the case of the EKF and the OF median values [13]. For this case, we do not take into account the IMU measurements. Figure 5a shows the estimated longitudinal velocity $V$ and steering angle $\varnothing$ after the FDE step. The GKLD residual used to quantify the divergence between the predicted and the updated distributions of the EIF is shown in figure $5 \mathrm{~b}$. Figure $5 \mathrm{c}$ shows the errors in position and orientation when applying the EIF with the FDE step (dark solid line) compared to the case of the Extended Kalman Filter (EKF) and using the OF median values (light solid line). The amelioration obtained in the case of the proposed informational framework is remarkable. Indeed, the mean position error drops from $0.3731 \mathrm{~m}$ when using the EKF with the OF median value to $0.2953 \mathrm{~m}$ when using the EIF with the FDE step.

A summary of the error values between the estimated travelled distance and steering angle and the ground truth in different cases can be found in Table 1. One should notice that the mean errors when fusing the IMU with the OF measurements are higher than the case of fusing only the OF measurements because of the large perturbations present in the IMU measurements in this case. The purpose of this fusion is therefore to find a method that takes into account all the available measurements while excluding automatically the erroneous one. It should be noted that the OF measurements are not available permanently.

Another test is done outdoor using various ground textures where four areas with different textures are placed on the floor. The robot is driven on a circular path at a velocity ranging from 0.4 to $0.8 \mathrm{~m} / \mathrm{s}$. In a similar way to the Figure 4 , Figure 6 shows the performance of the informational framework after fusing the OF with the IMU measurements. The mean position error which is 1.7776 before the FDE step drops to 0.4672 after the FDE step. Likewise, the mean orientation error drops from 3.0427 before the FDE step to 0.3865 after the FDE step.

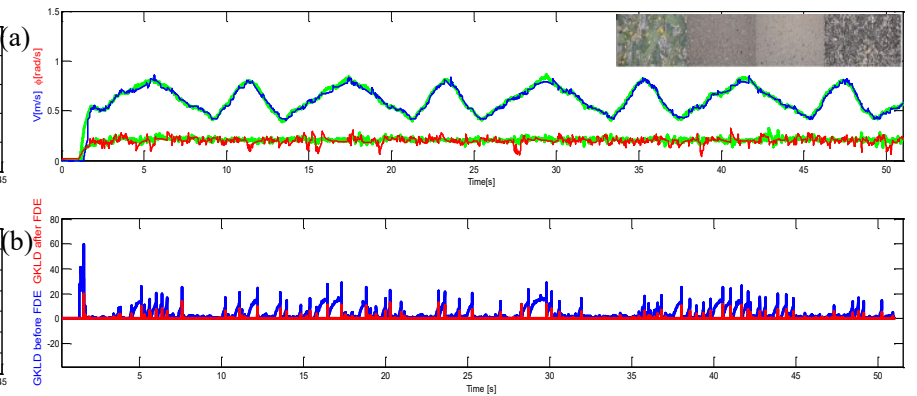

(c)

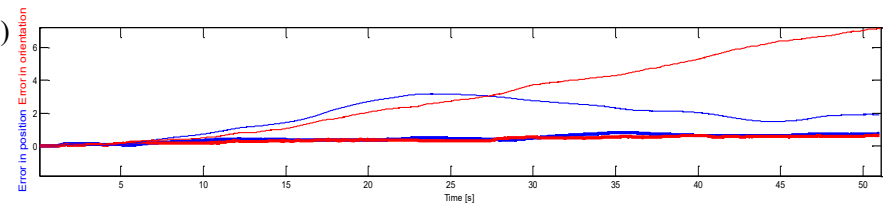

(d)

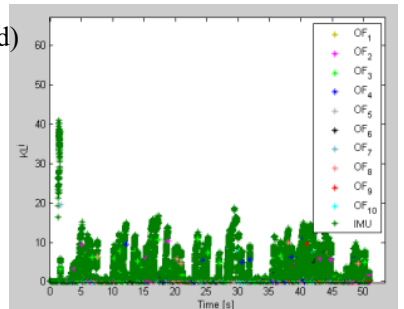

(e)

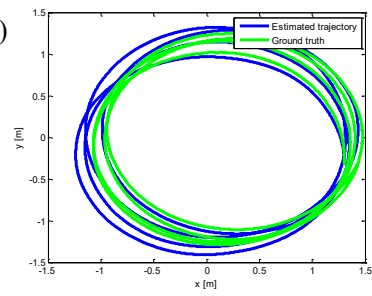

Fig. 6. Results obtained outdoors when the robot was driven on a circular path on various ground textures after fusing the IMU measurements with the OF data. (a) The estimated state vector after the FDE step (in blue and red), versus the ground truth values (in green). (Insert) Picture of the ground texture during the trial. (b) The $G K L D$ residual used for the fault detection (before the FDE step in blue, after the FDE step in red). (c) The error in the absolute position and orientation estimation before the FDE step (light solid line) and after the FDE step (dark solid line). (d) The set of residuals used for the fault exclusion. (e) The estimated trajectory after the FDE step (in blue) compared to reference trajectory (in green). 
(a)

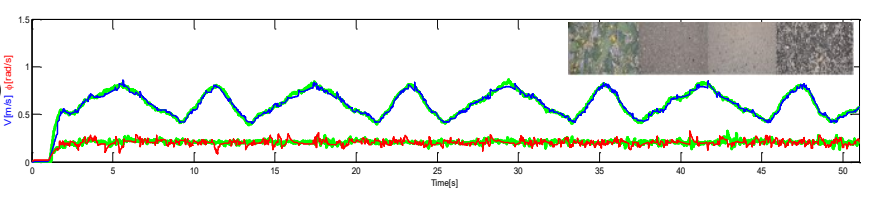

(b)

(c)

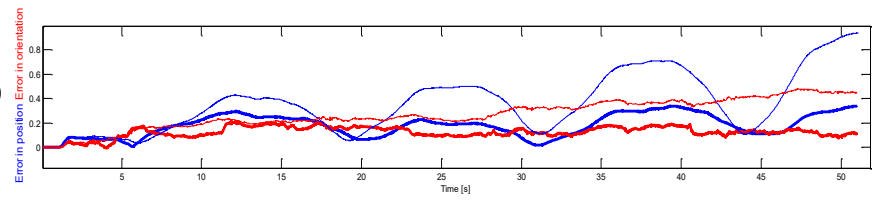

Fig. 7. Results without IMU obtained outdoors when the robot was driven on a circular path on various ground textures after fusing the OF raw data. (a) The estimated state vector after the FDE step (in blue and red), versus the ground truth values (in green). (Insert) Picture of the ground texture during the trial. (b) The GKLD residual used for the fault detection. (c) The error in the absolute position and orientation estimation after the FDE step (dark solid line) compared to the ones obtained using the EKF and the OF median value (light solid line).

(a)
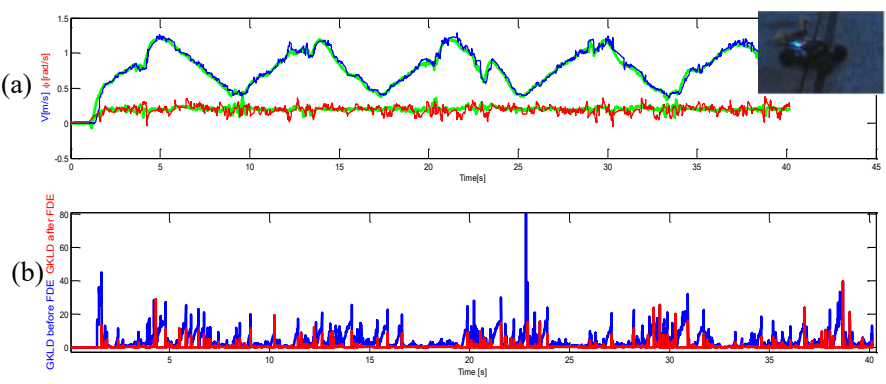

(c)

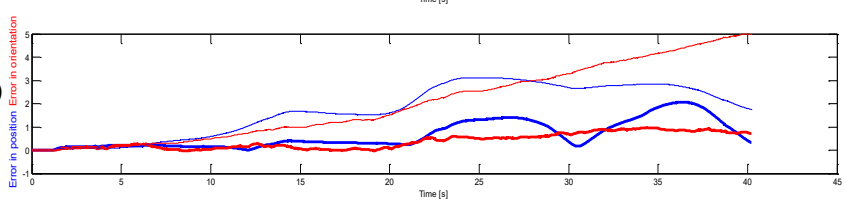

(d)

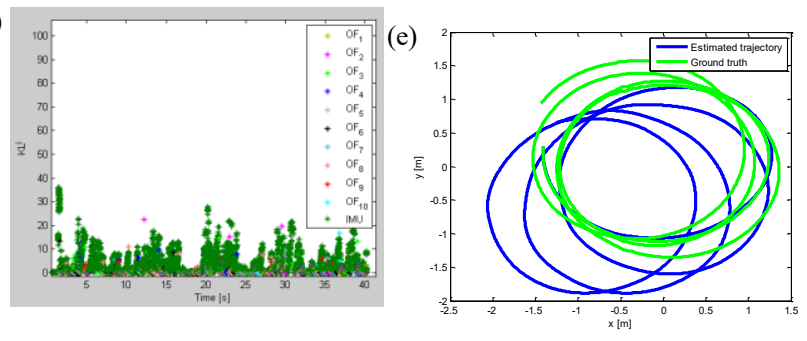

Fig. 8. Results obtained outdoors when the robot was driven at very low light in presence of recurrent vertically disturbance after fusing the IMU measurements with the OF data. (a) The estimated state vector after the FDE step. (Insert) Picture of the mobile robot during the trial. (b) The GKLD residual used for the fault detection. (c) The error in the absolute position and orientation estimation before the FDE step (light solid line) and after the FDE step (dark solid line). (d) The set of residuals used for the fault exclusion. (e) The estimated trajectory after the FDE step compared to reference trajectory.
Figure 7 shows the results after applying the proposed informational framework for fault tolerant data fusion when using OF raw data in the update step of the EIF without taking into account the IMU measurements. Comparison with the EKF that uses the OF median values is presented in Figure $7 \mathrm{c}$. The mean position error of 0.3568 obtained from the EKF goes to 0.1802 when using the EIF with the FDE step. Similarly, the mean orientation error of 0.2703 obtained from the EKF goes to 0.1251 when using the EIF.

A summary of the error values in different cases can be found in Table 2 .

The difference in results between the EKF and the EIF can be explained as follows. Indeed, to be robust to outliers, the approach developed with the EKF uses the median value of the 10 optical flow measurements without considering a consistency test. However, in the approach developed in this paper we test the consistency of the different OF measurements and the ones which pass the GKLD test are considered as valid measurements and then used for the state estimation. As noted in the paper, the FDE procedure is simple thanks to the update step of the EIF which is modelled as a simple summation of informational innovations.

Figure 8 shows the results for the same experiment presented in Figure 4 but when the robot is driven at very low light. The amelioration before and after the FDE step is remarkable as it appears in Figure 8c.

Table 1. A summary of the obtained errors values in different case when the robot was driven on a circular path in presence of recurrent vertically disturbance at very high light level (see scenario figures 4 and 5).

\begin{tabular}{|c|l|c|c|}
\hline \multicolumn{2}{|r|}{ Mean error in } & position & orientation \\
\hline \multirow{2}{*}{$\mathrm{E}$} & $\begin{array}{l}\text { Data fusion between IMU } \\
\text { and OF with FDE step }\end{array}$ & 0.7169 & 0.5531 \\
\cline { 2 - 4 } I & $\begin{array}{l}\text { Data fusion between IMU } \\
\text { and OF without FDE step }\end{array}$ & 1.7256 & 1.923 \\
\cline { 2 - 4 } $\mathrm{F}$ & $\begin{array}{l}\text { OF measurements data } \\
\text { fusion with FDE step }\end{array}$ & 0.2953 & 0.2155 \\
\cline { 2 - 4 } & $\begin{array}{l}\text { OF measurements without } \\
\text { FDE step }\end{array}$ & 0.3135 & 0.2265 \\
\hline $\mathrm{E}$ & $\begin{array}{l}\text { OF measurements using } \\
\mathrm{K}\end{array}$ & 0.3731 & 0.2922 \\
$\mathrm{nyyy} \mathrm{F}$ & IMU measurements & 1.5885 & 2.7386 \\
\hline
\end{tabular}

Table 2. A summary of the obtained errors values in different case when the robot was driven on a circular path on various ground textures (see scenario figures 6 and 7).

\begin{tabular}{|c|l|c|c|}
\hline \multicolumn{2}{|r|}{ Mean Error in } & position & orientation \\
\hline \multirow{4}{*}{$\begin{array}{l}\text { E } \\
\text { I }\end{array}$} & $\begin{array}{l}\text { Data fusion between IMU } \\
\text { and OF with FDE step }\end{array}$ & 0.4672 & 0.3865 \\
\cline { 2 - 4 } & $\begin{array}{l}\text { Data fusion between IMU } \\
\text { and OF without FDE step }\end{array}$ & 1.7776 & 3.0427 \\
\cline { 2 - 4 } & $\begin{array}{l}\text { OF measurements data } \\
\text { fusion with FDE step }\end{array}$ & 0.1802 & 0.1251 \\
\cline { 2 - 4 } & $\begin{array}{l}\text { OF measurements data } \\
\text { fusion without FDE step }\end{array}$ & 0.2190 & 0.1540 \\
\hline $\mathrm{E}$ & $\begin{array}{l}\text { OF measurements using } \\
\mathrm{K}\end{array}$ & 0.3568 & 0.2703 \\
\cline { 2 - 4 } $\mathrm{F}$ & IMU measurements & 1.6917 & 3.3177 \\
\hline
\end{tabular}




\section{CONCLUSION}

In this paper, an informational approach for ensuring the localization integrity of mobile robot is proposed. It is based on data fusion of OF and IMU measurements through the EIF for the state estimation and on the GKLD residual for the faults detection. For the exclusion of the faulty sensors measurements, a bank of EIF is developed and a set of residual $K L_{k}^{j}$ is generated. Each residual takes into account the information contributions of one measurement. The main advantage of this informational procedure is that will be able to detect multiple faults at a time with an advantageous processing time thanks to the modelling of the update step of the EIF as a simple sum of the information contributions of different observations. Moreover, the residual based on the KLD takes into account different kinds of information (Mahalanobis and Burg matrix divergence) between the predicted and the update distributions.

Experimental results carried out with varying speeds in outdoor environments show that the approach reacts suitably by excluding automatically the erroneous IMU measurements, strongly affected by the vibratory disturbance and the change in the ground texture. Likewise, the tests show the value of the $\mathrm{OF}$ sensors and their robustness to disturbances, which is greater than that of the IMU measurements. In addition, the performance of the OF sensors is independent from the light conditions.

Further odometry experiments including automatic threshold adjustment and an extended dynamical robotic model in closed loop is planned in a near future.

\section{ACKNOWLEDGEMENTS}

This research was supported partly by the CNRS (Life Science; Information and Engineering Science and Technology) and Aix-Marseille University. This research was also funded by a $\mathrm{PhD}$ grant from ANRT (Association Nationale de la Recherche et de la Technologie) as well as by PSA Peugeot Citroën via the OpenLab agreement with AixMarseille University and CNRS entitled "Automotive Motion Lab".

\section{REFERENCES}

[1] B. Khaleghi, A. Khamis, F. O. Karray, and S. N. Razavi, "Multisensor data fusion: A review of the stateof-the-art," Inf. Fusion, vol. 14, no. 1, pp. 28-44, 2013.

[2] A. Frikha and H. Moalla, "Analytic hierarchy process for multi-sensor data fusion based on belief function theory," Eur. J. Oper. Res., vol. 241, no. 1, pp. 133147, 2015.

[3] S. J. Wellington, J. K. Atkinson, and R. P. Sion, "Sensor validation and fusion using the Nadaraya-Watson statistical estimator," in Proceedings of the Fifth International Conference on Information Fusion, 2002, 2002, vol. 1, pp. 321-326 vol.1.

[4] D. M. Helmick, Y. Cheng, D. S. Clouse, L. H. Matthies, and S. I. Roumeliotis, "Path following using visual odometry for a mars rover in high-slip environments," in Aerospace Conference, 2004. Proceedings. 2004 IEEE, 2004, vol. 2, pp. 772-789.

[5] M. Maimone, Y. Cheng, and L. Matthies, "Two years of visual odometry on the mars exploration rovers," $J$. Field Robot., vol. 24, no. 3, pp. 169-186, 2007.

[6] R. L. Marks, H. H. Wang, M. J. Lee, and S. M. Rock, "Automatic visual station keeping of an underwater robot," in OCEANS '94. "Oceans Engineering for Today's Technology and Tomorrow's Preservation." Proceedings, 1994, vol. 2, p. II/137-II/142 vol.2.

[7] D. Scaramuzza, F. Fraundorfer, and R. Siegwart, "Realtime monocular visual odometry for on-road vehicles with 1-point RANSAC," in 2009 IEEE International Conference on Robotics and Automation, 2009, pp. 4293-4299.

[8] A. Zhu, N. Atanasov, and K. Daniilidis, "Event-based visual inertial odometry," in Proc. IEEE Int. Conf. Comput. Vis. Pattern Recog, 2017, vol. 3.

[9] A. R. Vidal, H. Rebecq, T. Horstschaefer, and D. Scaramuzza, "Ultimate SLAM? Combining Events, Images, and IMU for Robust Visual SLAM in HDR and High-Speed Scenarios," IEEE Robot. Autom. Lett., vol. 3, no. 2, pp. 994-1001, 2018.

[10] H. Alismail, M. Kaess, B. Browning, and S. Lucey, "Direct visual odometry in low light using binary descriptors," IEEE Robot. Autom. Lett., vol. 2, no. 2, pp. 444-451, 2017.

[11] M. Dille, B. Grocholsky, and S. Singh, "Outdoor downward-facing optical flow odometry with commodity sensors," in Field and Service Robotics, 2010, pp. 183-193.

[12] I. Nagai, K. Watanabe, K. Nagatani, and K. Yoshida, "Noncontact position estimation device with optical sensor and laser sources for mobile robots traversing slippery terrains," in Intelligent Robots and Systems (IROS), 2010 IEEE/RSJ International Conference on, 2010, pp. 3422-3427.

[13] S. Mafrica, A. Servel, and F. Ruffier, "Minimalistic optic flow sensors applied to indoor and outdoor visual guidance and odometry on a car-like robot," Bioinspir. Biomim., vol. 11, no. 6, p. 066007, 2016.

[14] H. Durrant-Whyte, Introduction to decentralised data fusion. The University of Sydney, Sydney, Australia, 2004.

[15] J. Al Hage, M. E. El Najjar, and D. Pomorski, "Multisensor fusion approach with fault detection and exclusion based on the Kullback-Leibler Divergence: Application on collaborative multi-robot system," Inf. Fusion, vol. 37, pp. 61-76, Sep. 2017.

[16] S. I. Roumeliotis, G. Sukhatme, and G. A. Bekey, "Sensor fault detection and identification in a mobile robot," in Proceedings in International Conference on Intelligent Robots and Systems, 1998., 1998, vol. 3, pp. 1383-1388.

[17] A.-C. Escher, C. Macabiau, N. Martin, B. Roturier, and V. Vogel, "GNSS/IRS hybridization: fault detection and isolation of more than one range failure," in ION GPS 2002, 15th International Technical Meeting of the Satellite Division of The Institute of Navigation, 2002, p. 2619. 
[18] T. Wei, Y. Huang, and P. Chen, "Sensor validation for flight control by particle filtering," in Signal Processing Conference, 2005 13th European, 2005, pp. 1-4.

[19] M. Kumar, D. P. Garg, and R. A. Zachery, "A Method for Judicious Fusion of Inconsistent Multiple Sensor Data," IEEE Sens. J., vol. 7, no. 5, pp. 723-733, May 2007.

[20] V. Ricquebourg, M. Delafosse, L. Delahoche, B. Marhic, A. M. Jolly-Desodt, and D. Menga, "Fault detection by combining redundant sensors: a conflict approach within the tbm framework," Cogn. Syst. Interact. Sens. COGIS, 2007.

[21] A. De Luca, G. Oriolo, and C. Samson, "Feedback control of a nonholonomic car-like robot," Robot Motion Plan. Control, pp. 171-253, 1998.

[22] N. Assimakis, M. Adam, and A. Douladiris, "Information filter and kalman filter comparison: Selection of the faster filter," Int. J. Inf. Eng., vol. 2, no. 1, pp. 1-5, 2012.

[23] S. Kullback, Information theory and statistics. Courier Corporation, 1968.

[24] J. V. Davis and I. S. Dhillon, "Differential Entropic Clustering of Multivariate Gaussians," in Neural information processing systems (NIPS), 2006, pp. 337344.

[25] B. Kulis, M. Sustik, and I. Dhillon, "Learning low-rank kernel matrices," in Proceedings of the 23rd international conference on Machine learning, 2006, pp. 505-512.

[26] F. Roubieu, F. Expert, G. Sabiron, and F. Ruffier, "A two-directional 1-gram visual motion sensor inspired by the fly's eye," IEEE Sens. J., vol. 13, no. 3, pp. 10251035, 2013.

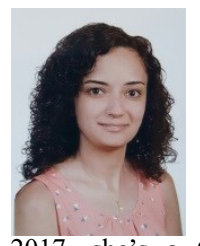

Joelle Al Hage received the engineer degree in electrical and electronic engineering and the master degree in Reliability, Identification and Diagnosis from the Lebanese university in 2013. She received the Ph.D. degree in automatic and computer science from university of Lille, France in 2016. In 2016/2017, she was an assistant lecturer and researcher at the University of Lille. Since October 2017, she's a CNRS young researcher at SIVALab laboratory (shared laboratory between Renault/Heudiasyc/CNRS) of the Sorbonnes universités, university of Technologie of Compiègne, France. Her research is focused on the multi-sensor data fusion with fault tolerance aspect based on an informational formalism and on the integrity of localization and perception methods for autonomous vehicle.

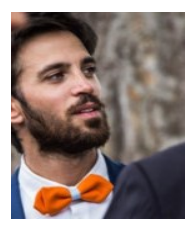

Stefano Mafrica received a BS degree in Information Technologies Engineering from University of Genoa in 2010, a MS degree in Robotics Engineering from University of Genoa and Ecole Centrale de Nantes in 2012, and a Ph.D. degree in Biorobotics Engineering from Aix-Marseille University in 2016. He currently works at PSA Groupe as Innovation Lead Engineer in the field of Advanced Driver Assistance Systems and Autonomous Driving. His main areas of interest are perception, sensor fusion, decision and control of robotic systems.

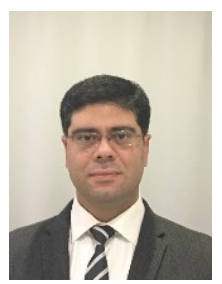

Maan El Badaoui El Najjar received the computer engineering degree and the Master of Science degree from the INP Grenoble - France in control system respectively in 1999 and in 2000 . He received the Ph.D. degree in Perception and Control Systems from the University of Technology of Compiegne in 2003. In November 2011, he obtained the Habilitation to Supervise Research (HDR) from the University of Lille. He joined the University of Lille in 2005 as a permanent associate Professor. Since 2014, he is Full Professor at the University of Lille. $\mathrm{He}$ is also the head of the DiCOT Team "Diagnostic, Control and Observation for fault Tolerant Systems" of the CRIStAL Laboratory UMR CNRS 9189. His research has been focused on fault tolerant multi-sensor fusion approach which is able to effectively deal with faulty sensors of dynamic systems and has desired methodological capacities for robotics and intelligent vehicle system applications.

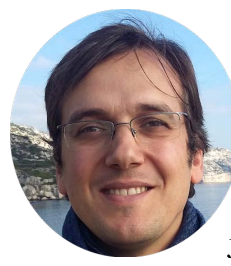

Franck Ruffier received an engineering degree in 2000 and a Ph.D. degree from INP-Grenoble in 2004, as well as a habilitation to supervise research (HDR in French) from Aix-Marseille University in 2013. He was visiting scientist invited by Prof. Michael Dickinson, Univ. of Washington, Seattle, USA during 2 months in 2012 as well as in 2008 by Dr. T. Mukai at RIKEN, Nagoya, Japan. Franck Ruffier published more than 58 papers in international Journals and referred Proceedings (Web of Science) as well as 12 book chapters and he filed 9 patents. His present position is CNRS research scientist at the Institute of Movement Science (ISM). His main areas of interest are insect vision and behavior as well as bio-inspired vision and robotics 